\title{
Damping and electromechanical energy losses in the piezoelectric polymer PVDF
}

\section{Authors: A. M. Vinogradov, V. Hugo Schmidt, G. F. Tuthill, and G. W. Bohannan}

NOTICE: this is the author's version of a work that was accepted for publication in Mechanics of Materials. Changes resulting from the publishing process, such as peer review, editing, corrections, structural formatting, and other quality control mechanisms may not be reflected in this document. Changes may have been made to this work since it was submitted for publication. A definitive version was subsequently published in Mechanics of Materials, VOL\# 36, ISSUE\# 10, (October 2004), DOI\# 10.1016/j.mechmat.2003.04.002

A.M. Vinogradov, V.H. Schmidt, G.F. Tuthill, and G.W. Bohannan, "Damping and electromechanical energy losses in the piezoelectric polymer PVDF," Mechanics of Materials 36, 1007-1016 (2004). doi: 10.1016/j.mechmat.2003.04.002 


\title{
Damping and electromechanical energy losses in the piezoelectric polymer PVDF
}

\author{
Aleksandra M. Vinogradov ${ }^{\mathrm{a}, *}$, V. Hugo Schmidt ${ }^{\mathrm{b}}$, George F. Tuthill ${ }^{\mathrm{b}}$, \\ Gary W. Bohannan ${ }^{\mathrm{c}}$ \\ ${ }^{a}$ Department of Mechanical and Industrial Engineering, Montana State University, Bozeman, MT 59717, USA \\ b Department of Physics, Montana State University, Bozeman, MT 59717, USA \\ ${ }^{\mathrm{c}}$ Wavelength Electronics, Bozeman, MT 59717, USA
}

Received 10 September 2002; received in revised form 3 March 2003

\begin{abstract}
Polyvinylidene fluoride (PVDF) is a piezoelectric polymer that has been used in many applications including microphones, transducers, sensors and actuators. The electromechanical properties of PVDF are commonly defined by the constitutive equations of piezoelectricity. This paper presents experimental evidence that the assumptions underlying the theory of piezoelectricity have certain limitations in terms of representing adequately the electromechanical properties of PVDF. It is shown that PVDF tends to demonstrate time-dependent behavior in the form of viscoelastic creep and dielectric relaxation, and measurable energy losses under cyclic loading conditions. Moreover, the response of PVDF strongly depends on temperature and cyclic frequencies.
\end{abstract}

(C) 2003 Elsevier Ltd. All rights reserved.

Keywords: Polyvinylidene fluoride; PVDF; Piezoelectric polymer; Electromechanical properties; Dielectric relaxation; Viscoelastic creep

\section{Introduction}

In recent years, a new generation of so-called "smart" materials has emerged that can detect changes in the loading or environmental conditions, choose rationally among a set of respective responses, and carry out these actions in a controlled manner. A broad range of applications utilizing such functions includes active vibration damping, acoustic suppression, damage detection, shape and position control of compliant structures, and self-inspection of structural integrity.

As an integral part of the "smart" materials group, piezoelectric polymers exhibit a type of behavior that is often compared with biological reactions involving transformations of the sensed information into the desired response. Due to such special qualities, piezoelectric polymers have been increasingly used in a rapidly expanding range of applications such as electromechanical transducers, position sensors and vibration control actuators. 
Many synthetic polymers, including polypropylene, polystyrene and poly(methyl methacrylate); semi-crystalline polyamides and amorphous polymers such as vinyl acetate have demonstrated piezoelectric properties. However, piezoelectric effects in these materials are relatively weak, often unstable and, therefore, are of limited practical significance. Strong and stable piezoelectric properties have been observed only in the synthetic polymer polyvinylidene fluoride $\left(\mathrm{PVDF}\right.$ or $\mathrm{PVF}_{2}$ ) and PVDF copolymers.

Polyvinylidene fluoride is a semi-crystalline polymer with typical crystallinity of $50 \%$. The amorphous phase of the polymer has the properties of a supercooled liquid with the glass transition temperature of about $-50{ }^{\circ} \mathrm{C}$. The molecular structure of polyvinylidene fluoride consists of the repeated monomer unit $-\mathrm{CF}_{2}-\mathrm{CH}_{2}-$. The atoms are covalently bonded together, forming long molecular chains. Since the hydrogen atoms are positively charged and the fluoride atoms are negatively charged with respect to the carbon atoms, PVDF is inherently polar if the molecular chains are aligned as in the $\beta$-phase.

Currently, polyvinylidene fluoride (PVDF) is the principal commercially available piezoelectric polymer, which is typically produced in the form of thin films of thickness ranging from 10 to 760 $\mu \mathrm{m}$. A thin layer of nickel, copper or aluminum is deposited on both surfaces of the material in order to provide electrical conductivity for applying an electrical field, or to allow measurements of the charge induced by mechanical deformations.

The response of PVDF and its copolymers is time-dependent and is characterized by electromechanical energy losses. For example, energy dissipation in PVDF under cyclic conditions has been observed through hysteresis loops formed by the measured dielectric displacement as function of the applied electric field (Furukawa et al., 1980; Gookin et al., 1984). Similar behavior has been observed in PVDF copolymers, vinylidene fluoride-trifluoroethylene (VDF-TrFE) and vinylidene fluoride-tetrafluoroethylene (VDF-TFE) (Furukawa et al., 1981; Davis et al., 1984). Piezoelectric, dielectric and mechanical relaxation of piezoelectric polymers has been investigated in a number of studies (Wang et al., 1993; Koga and
Ohigashi, 1986; Osaki and Kotaka, 1981; Arisawa et al., 1981). Complex elastic, dielectric and piezoelectric characteristics of poled PVDF films in the frequency domain have been determined experimentally by Roh et al. (2002).

The main objective of this paper is to investigate the time-dependent electrical and mechanical responses of PVDF thin films under various loading and temperature conditions. This work has been undertaken in conjunction with a NASA sponsored research project directed towards the development of a PVDF actuator for active vibration control in microgravity environments. In Sections 2 and 3 of the paper we report the results of two independent studies describing the properties of PVDF. In Section 4, we discuss our conclusions and offer suggestions for future research.

\section{Mechanical creep and relaxation of PVDF}

Since the discovery of piezoelectric effects in PVDF (Kawai, 1969), the properties of the polymer have been studied extensively. However, research efforts in this regard have focused mainly on the piezoelectric response of the polymer, whereas the mechanical behavior of PVDF has attracted limited attention (Vinogradov, 2002). In this study, the mechanical properties of PVDF have been characterized through a consistent experimental program, involving static tests, quasi-static creep tests and dynamic oscillatory tests of $28 \mu \mathrm{m}$ thick commercially produced PVDF thin films.

Stress-strain experiments have been performed under displacement-controlled tensile stress conditions at a $1.27 \mathrm{~cm} / \mathrm{min}$ strain rate. The respective measurements were recorded by an automatic data acquisition system. These tests have demonstrated that the mechanical response of poled PVDF films depends on the direction of the applied load in relation to the orientation of the aligned molecular chains of the polymer. In this regard it is important to note that the permanent polarization of PVDF is usually obtained through a process that involves stretching and poling of extruded thin sheets of PVDF. Stretching provides the alignment of molecular chains in the stretch direction. An 
applied electric field of up to $100 \mathrm{kV} / \mathrm{mm}$ at an elevated (typically $103{ }^{\circ} \mathrm{C}$ ) temperature causes a polarization that is maintained after the material is cooled to room temperature.

Based on stress-strain tests it has been observed that the response of PVDF thin films in the direction of the aligned molecular chains (direction 1) was characterized by a continuous increase of stress-strain curve that culminated in a sudden failure of material samples. Such response is typically observed in brittle materials. In contrast, in the direction normal to the orientation of the aligned molecular chains (direction 2) the respective stress-strain diagram was characteristic of ductile material behavior, involving an increase of stresses up to a certain maximum value with a following sharp decrease of the load carrying capacity of the sample (Vinogradov and Holloway, 1999).

Further, quasi-static creep experiments of 28 $\mu \mathrm{m}$ PVDF samples were performed in both material directions at 10 different stress levels under sustained loading conditions at room temperature. Strain measurements were taken using a linear variable differential transformer (LVDT) equipped with an automated data acquisition system. These creep tests established that at stress levels below $0.57\left(\sigma_{\mathrm{Y} 1}\right)$ and $0.76\left(\sigma_{\mathrm{Y} 2}\right)$ in the directions 1 and 2 , respectively, the creep behavior of PVDF was stress-independent within the margins of experimental accuracy. Note that $\sigma_{\mathrm{Y} 1}=45 \mathrm{MPa}$ and $\sigma_{\mathrm{Y} 2}=39 \mathrm{MPa}$ are the yield stresses of PVDF in the directions 1 and 2, respectively. Within these limits, the creep compliances of PVDF have been obtained in both material directions, as shown in Figs. 1 and 2.

The creep behavior of PVDF thin films has been characterized by the constitutive equations of linear viscoelasticity in the form (Wineman and Rajagopal, 2000)

$\varepsilon(t)=\sigma(0) C_{n}(t)+\int_{0}^{t} C_{n}(t-\tau) \frac{\mathrm{d} \sigma}{\mathrm{d} \tau} \mathrm{d} \tau$,

where the stress $\sigma$ and strain $\varepsilon$ are functions of time $t$, and $C_{n}(n=1,2)$ denote the creep compliances in the respective material direction of PVDF.

The creep compliances $C_{n}(t)$ of PVDF shown in Figs. 1 and 2 can be represented analytically in the form of a power law

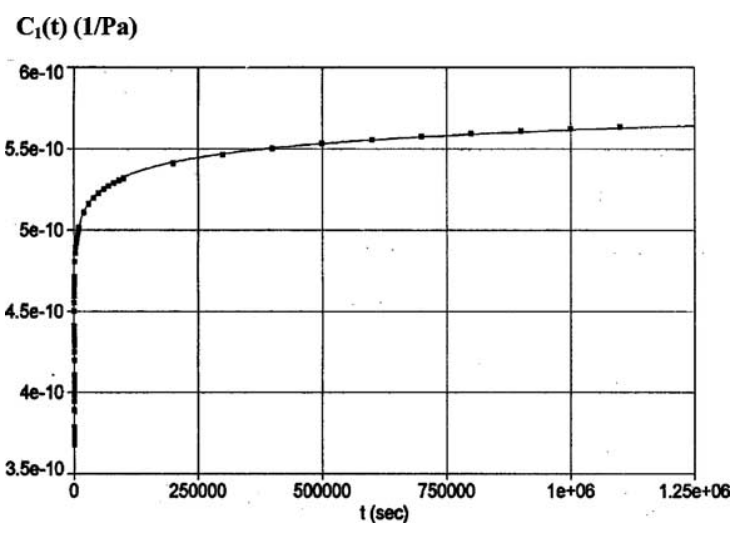

Fig. 1. Creep compliance of PVDF (material direction 1).

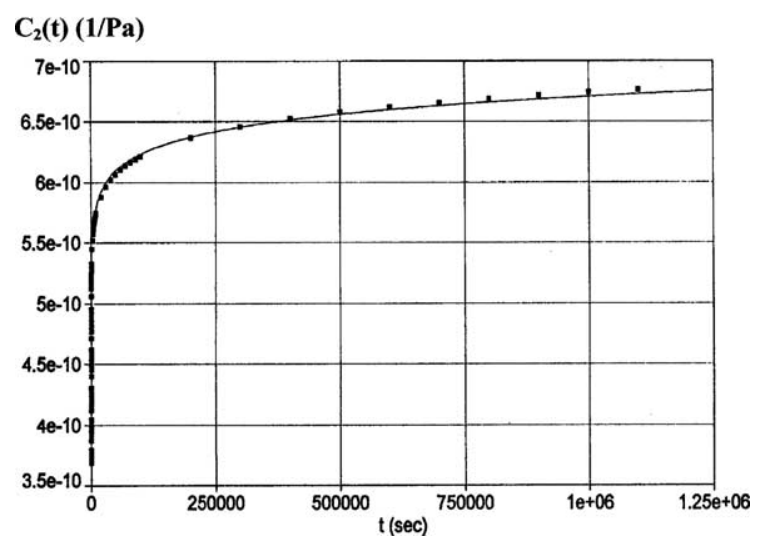

Fig. 2. Creep compliance of PVDF (material direction 2).

$C_{n}(t)=a_{n}+b_{n}\left(t \alpha_{n}\right), \quad n=1,2$,

where the coefficients $a_{n}, b_{n}$ and $\alpha_{n}$ are as follows:

Direction 1: $a_{1}=3.206 \times 10^{-10}$;

$$
\begin{aligned}
& b_{1}=5.018 \times 10^{-11} ; \\
& \alpha_{1}=0.107,
\end{aligned}
$$

Direction 2: $a_{2}=3.514 \times 10^{-10}$;

$$
\begin{aligned}
& b_{2}=0.111 \times 10^{-11} ; \\
& \alpha_{2}=0.085 .
\end{aligned}
$$

Substitution of Eq. (2) into Eq. (1) defines the creep properties of PVDF in the form 


$$
\begin{aligned}
\varepsilon(t) & =a_{n} \sigma(t)+b_{n} C_{n} \int_{0}^{t}\left[\sigma(\tau) /(t-\tau)^{1-\alpha_{n}}\right] \mathrm{d} \tau, \\
n & =1,2 .
\end{aligned}
$$

The mechanical relaxation of PVDF in both material directions can be characterized by solving Eq. (5) in terms of $\sigma(t)$. Note that Eq. (5) is known as the Abel equation and can be solved using the Laplace transformation method. The solution is of form (Kanwal, 1971):

$$
\begin{aligned}
\sigma(t) & =\frac{\varepsilon(t)}{a_{n}}-\frac{1}{a_{n}} \int_{0}^{t} \sum_{n=1}^{\infty} \frac{\left(b_{n} \alpha_{n} / a_{n}\right) \Gamma\left(\alpha_{n}\right)(t-\tau)^{\alpha_{n}}}{(t-\tau) \Gamma\left(n \alpha_{n}\right)} \varepsilon(\tau) \mathrm{d} \tau, \\
n & =1,2,
\end{aligned}
$$

where $\Gamma\left(\alpha_{n}\right)$ and $\Gamma\left(n \alpha_{n}\right)$ are gamma functions.

The latter equation describes the stress relaxation of the material for any given strain history $\varepsilon(t)$. In particular, at a constant strain $\varepsilon_{0}$, Eq. (6) assumes the form

$$
\begin{aligned}
\frac{\sigma(t)}{\varepsilon_{0}} & =\frac{1}{a_{n}}\left[1-\int_{0}^{t} \sum_{n=1}^{\infty} \frac{\left(b_{n} \alpha_{n} / a_{n}\right) \Gamma\left(\alpha_{n}\right)(t-\tau)^{\alpha_{n}}}{(t-\tau) \Gamma\left(n \alpha_{n}\right)} \mathrm{d} \tau\right] \\
& =\frac{1}{a_{n}}\left[1-R_{n}(t)\right], \quad n=1,2
\end{aligned}
$$

where $R_{n}(t) \quad(n=1,2)$ represents the relaxation function of PVDF in both in-plane material directions.

Based on Eq. (7), a graphical representation of the relaxation functions $R_{n}(t)$ in both in-plane directions of PVDF was obtained using the creep parameters specified by Eqs. (3) and (4). The respective diagrams are shown in Figs. 3 and 4.

In this study, a consistent dynamic testing program was also developed to determine the mechanical response of PVDF under cyclic loading conditions. Time-dependent mechanical properties of PVDF were characterized in terms of the complex modulus $Y^{*}=Y^{\prime}+\mathrm{i} Y^{\prime \prime}$ and complex creep compliance $C^{*}=C^{\prime}+\mathrm{i} C^{\prime \prime}$ where $Y^{\prime}$ and $Y^{\prime \prime}$ denote the storage and loss moduli; $C^{\prime}$ and $C^{\prime \prime}$ denote the storage and loss compliances, respectively. These quantities allow the characterization of the material's ability to store energy $\left(Y^{\prime}, C^{\prime}\right)$ or dissipate energy $\left(Y^{\prime \prime}, C^{\prime \prime}\right)$. The mechanical damping properties of a viscoelastic material are typically

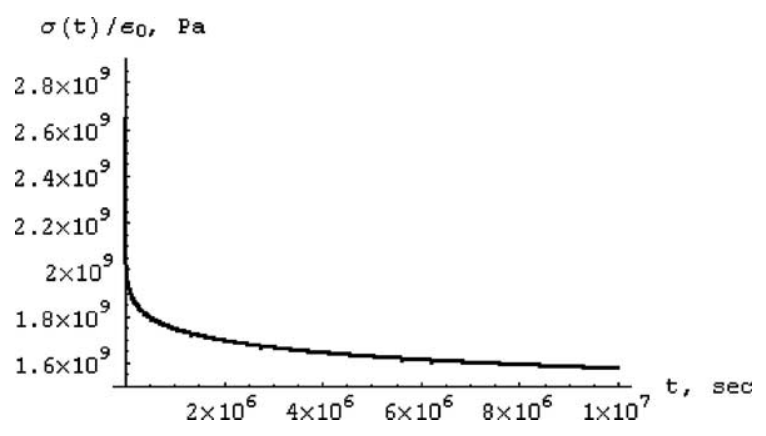

Fig. 3. Mechanical relaxation function of PVDF (material direction 1).

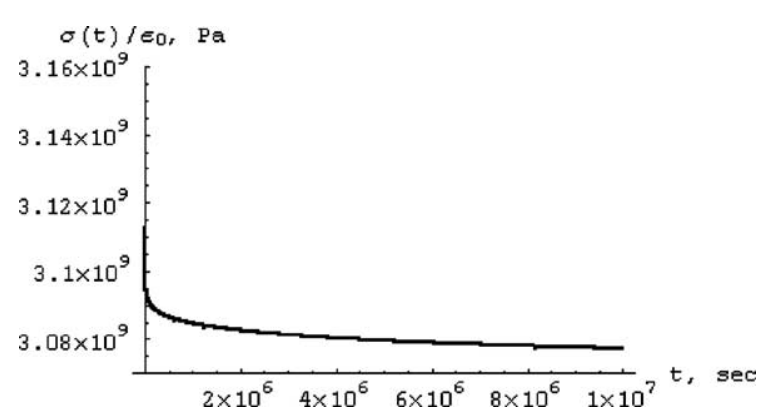

Fig. 4. Mechanical relaxation function of PVDF (material direction 2).

defined in terms of the ratio $Y^{\prime \prime} / Y^{\prime}=\tan \delta(\mathrm{Me}-$ nard, 1999).

To determine these characteristics of PVDF, a dynamic experimental program was developed involving a series of oscillatory tests. In the experiments, PVDF samples were subjected to sustained tensile stress at several levels within linear viscoelastic limits, plus a superimposed sinusoidal strain $\varepsilon=\varepsilon_{a} \sin (\omega t)$. The response of the material was measured in terms of the respective sinusoidal stress $\sigma=\sigma_{a} \sin (\omega t+\delta)$, with the phase shift $\delta$ representing the loss of mechanical energy, as defined above.

The dynamic tests were performed in each material direction of PVDF samples at 20 different frequencies ranging from $\omega=1-50 \mathrm{~Hz}$ under ambient temperature conditions. The experimental frequency range was further expanded on the basis of the temperature-frequency correspondence principle (Ferry, 1980) that provides a relation 
between the viscoelastic material characteristics at a reference temperature $T_{0}$ and the respective material characteristics at any other temperature $T$ such that

$Y^{\prime}(\omega, T)=Y^{\prime}\left(\omega a_{T}, T_{0}\right)$,

where $a_{T}$ denotes the shift factor which depends on temperature.

For PVDF thin films, the shift factor $a_{T}$ was determined by repeating the entire set of dynamic experiments at 13 different temperature levels in the range from 25.5 to $81^{\circ} \mathrm{C}$ for material direction 1 , and 10 different temperature levels in the range from 24.4 to $81.1{ }^{\circ} \mathrm{C}$ for material direction 2. On this basis, the loss and storage moduli $Y^{\prime}$ and $Y^{\prime \prime}$ were determined as functions of temperature and frequency, as shown in Figs. 5 and 6. Similar

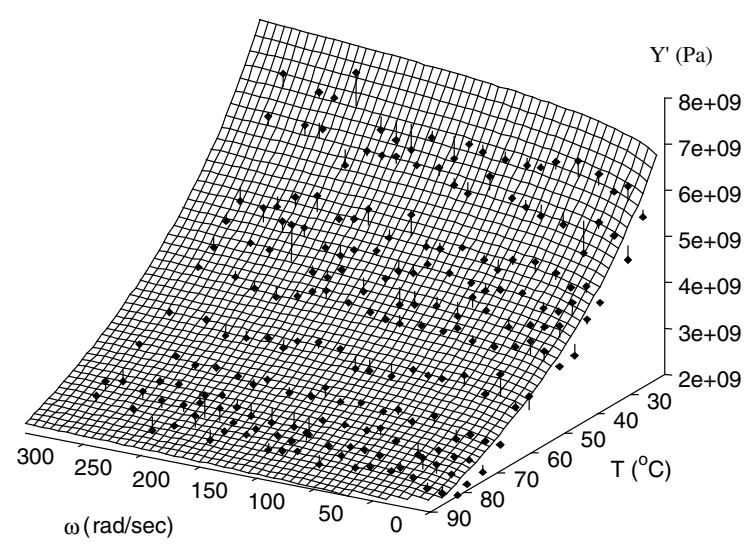

Fig. 5. Storage modulus of PVDF (material direction 1).

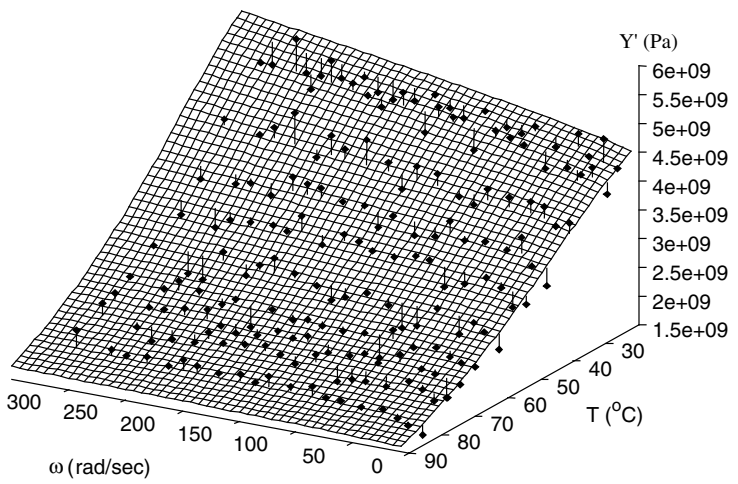

Fig. 6. Storage modulus of PVDF (material direction 2). relations have been obtained for the storage and loss compliances of PVDF (Holloway, 1997).

The experimental results obtained from the dynamic testing program were used to validate the linear viscoelastic constitutive model of PVDF as determined from the quasi-static creep experiments. This goal was accomplished by inverse Fourier transformation of the storage and loss moduli and the storage and loss compliances obtained in the frequency domain from the dynamic experiments. Using the approximate method by Ninomiya and Ferry (Ferry, 1980), the relaxation functions $R_{n}(t)$ and creep compliances $C_{n}(t)$ in two in-plane material directions of PVDF were obtained from the relations

$$
\begin{aligned}
R_{n}(t)= & Y_{n}^{\prime}(\omega)-0.40 Y_{n}^{\prime \prime}(0.40 \omega) \\
& +\left.0.014 Y_{n}^{\prime \prime}(10 \omega)\right|_{\omega=1 / t}, \\
C_{n}(t)= & C_{n}^{\prime}(\omega)+0.40 C_{n}^{\prime \prime}(0.40 \omega) \\
& -\left.0.014 C_{n}^{\prime \prime}(10 \omega)\right|_{\omega=1 / t}
\end{aligned}
$$

The results have confirmed the accuracy of the creep and relaxation characteristics of PVDF determined from the quasi-static creep experiments within the range of linear viscoelasticity.

\section{Dielectric relaxation in PVDF}

A second goal of this research was to create a dynamic constitutive model of polarization, expressed as a time domain differential equation. Our aim was to develop a model that can predict the long-time-scale power-law relaxation seen in virtually all dielectric materials. An ultimate use of the electrical and mechanical models is to develop a combined actuator model for use in vibration and position control systems. Moreover, in this particular study we wished to compare electrical and mechanical loss terms (as represented by power-law exponents) over the same time scales. This required developing a model for dielectric relaxation that allows extrapolation from short time scales (where ac dielectric measurements can be reliably made) to the much longer time scales that are the domain of the mechanical studies reported in Section 2. 
The method chosen to guide this development was to generalize an existing model, consisting of ideal circuit elements, to the power-law form of fractional calculus. This approach was motivated by Nonnenmacher and Glockle (1991), who suggested applying fractional calculus to the problem of mechanical relaxation in polymers. In dielectric relaxation the decay current (i.e. the time rate of change of the electric displacement) is measured rather than the electric displacement itself, as in mechanical systems; otherwise, the approaches are equivalent. In this case, the relaxation currents reflect the growth or decay of the charge (or polarization) in the material, which is the electrical analog of the mechanical creep. Our fractional calculus approach can also be cast in a form (Oldham and Spanier, 1974) analogous to that of the Abel integral shown in Eq. (5) for the mechanical response.

An equivalent circuit model, such as shown in Fig. 7, may be taken as an initial candidate for describing a "lossy" dielectric material. Other models can be used, leading to slightly different forms of the constitutive equation but producing very similar predictions of long-time behavior. The impedance of the circuit in Fig. 7 is:

$$
Z(\omega)=\frac{1}{\mathrm{i} \omega C_{1}+\frac{1}{R_{1}}+\frac{1}{R_{2}+\frac{1}{\mathrm{i} \omega C_{2}}}},
$$

which can be transformed to a permittivity $\varepsilon(\omega)=\varepsilon^{\prime}(\omega)+\mathrm{i} \varepsilon^{\prime \prime}(\omega)$, according to

$\varepsilon(\omega)=\frac{1}{\mathrm{i} \omega C_{\mathrm{C}} Z(\omega)}$,

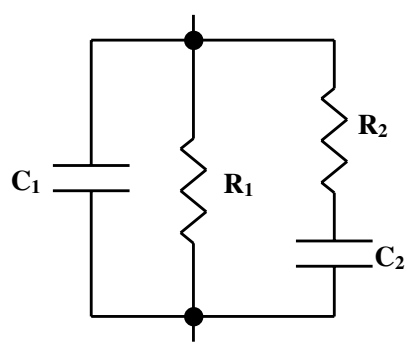

Fig. 7. An ideal element circuit incorporating conductivity. where $C_{\mathrm{C}}=\varepsilon_{0} \times$ area/depth is the capacitance of the empty test cell, to obtain the dielectric function

$\varepsilon(\omega)=\frac{\frac{C_{1}+C_{2}}{C_{\mathrm{C}}}+\frac{\tau}{R_{1} C_{\mathrm{C}}}+\frac{C_{1}}{C_{\mathrm{C}}}(\mathrm{i} \omega \tau)+\frac{R_{2}}{R_{1}} \frac{C_{2}}{C_{1}}(\mathrm{i} \omega \tau)^{-1}}{1+\mathrm{i} \omega \tau}$,

where $\tau=R_{2} C_{2}$. By replacing the resistive elements with constant phase elements (CPE) according to the prescription of Cole and Cole (1941):

$R_{1} \rightarrow R_{1}(\mathrm{i} \omega \tau)^{-\gamma}, \quad R_{2} \rightarrow R_{2}(\mathrm{i} \omega \tau)^{-\alpha}$,

we obtain

$\varepsilon(\omega)=\frac{\varepsilon_{\mathrm{S}}+\varepsilon_{\infty}(\mathrm{i} \omega \tau)^{\delta}+\varepsilon_{\mathrm{C}}(\mathrm{i} \omega \tau)^{\beta}+\varepsilon_{\mathrm{C}}(\mathrm{i} \omega \tau)^{v}}{1+(\mathrm{i} \omega \tau)^{\delta}}$

for the frequency-dependent dielectric permittivity, where

$\tau=R_{2} C_{2}, \quad \varepsilon_{\mathrm{S}}=\frac{C_{1}+C_{2}}{C_{\mathrm{C}}}, \quad \varepsilon_{\infty}=\frac{C_{1}}{C_{\mathrm{C}}}$,

$\varepsilon_{\mathrm{C}}=\frac{\tau}{R_{1} C_{\mathrm{C}}}, \quad \delta=1-\alpha, \quad \beta=\gamma-1, \quad v=\gamma-\alpha$.

The CPE represents a linear element with a frequency independent phase shift that lies between that of a resistor and that of a capacitor, i.e. $0<\gamma<1$ and $0<\alpha<1$. In more conventional language, the power law exponents account for the "distribution of time constants" that appear in dielectric relaxation. Further discussion of the concept of the constant phase element can be found in Cole and Cole (1941). The power-law "distribution of time constants" results in a flattening of the loss part of the permittivity curve, $\varepsilon^{\prime \prime}$ versus $\omega$, from that which would be expected from a dielectric obeying the Debye model, thereby sometimes earning the term "constant loss element," although the loss tangent, $\varepsilon^{\prime \prime} / \varepsilon^{\prime}$, is not strictly constant.

In these studies, we first made impedance measurements over the frequency range $20 \mathrm{~Hz}$ to 300 $\mathrm{kHz}$, on samples cut from a $28 \mu \mathrm{m}$ thick sheet of piezoelectric PVDF with silver electrode layers. The results were converted to the real and imaginary permittivity values shown in Fig. 8. The thermally activated resonance is an electromechanical response that will be ignored in what follows. These 


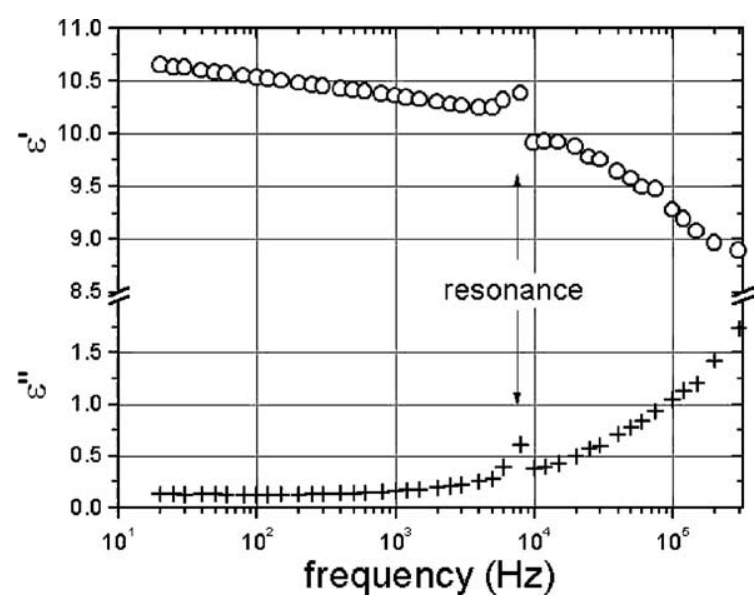

Fig. 8. Real and imaginary parts of the permittivity of a $28 \mu \mathrm{m}$ thick sample of PVDF.

data were then fit to Eq. (5) with the results: $\tau=152$ ns, $\delta=0.65, \quad \beta=-0.085, \quad v=0.565, \quad \varepsilon_{\mathrm{S}}=9.3$, $\varepsilon_{\mathrm{C}}=0.46, \varepsilon_{\infty}=2.7$. The Cole-Cole plot of the spectroscopic measurements, together with the fit (and extrapolation to low frequency) is shown in Fig. 9.

Our model can then used to predict timedependent behavior in the very long-time domain. In general, we can construct a form of the constitutive equation relating polarization to the applied field, by starting with the electric displacement,

$\mathbf{D}(t)=\varepsilon_{0} \mathbf{E}(t)+\varepsilon_{0} \int_{-\infty}^{\infty} f\left(t^{\prime}\right) \mathbf{E}\left(t-t^{\prime}\right) \mathrm{d} t^{\prime}$,

with $f(t)=0$ for $t<0$,

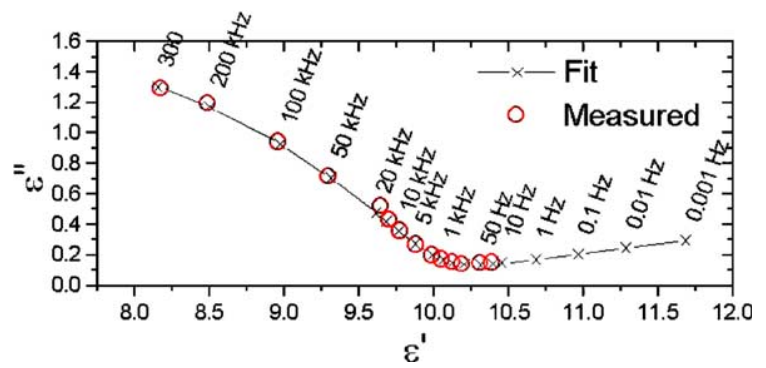

Fig. 9. Cole-Cole plot derived from the data of Fig. 8. The data were fitted to Eq. (15) with the values $\tau=152 \mathrm{~ns}, \delta=0.65$, $\beta=-0.085, \quad v=0.565, \quad \varepsilon_{\mathrm{S}}=9.3, \quad \varepsilon_{\mathrm{C}}=0.46, \quad \varepsilon_{\infty}=2.7$. The extrapolation to the very low frequency regime is shown at the right. where $f(t)$ represents a memory function associated with the material properties of the dielectric and $\mathbf{E}(t)$ is the applied electric field (Landau and Lifshitz, 1960). In the frequency domain we have $\mathbf{D}(\omega)=(1+\chi(\omega)) \varepsilon_{0} \mathbf{E}(\omega)$, so

$\chi_{\mathrm{e}}(\omega)=\varepsilon(\omega)-1=\int_{-\infty}^{\infty} f\left(t^{\prime}\right) \mathrm{e}^{\mathrm{i} \omega t^{\prime}} \mathrm{d} t^{\prime}$.

That is, $\chi_{\mathrm{e}}(\omega)$ is just the Fourier transform of the memory function.

To translate the dielectric function into a time domain differential equation, we interpret $\mathrm{i} \omega$ as the Laplace variable $s$, and make the operator association $s \rightarrow \mathrm{d} / \mathrm{d} t$. In this case, $(\mathrm{i} \omega \tau)^{q} \rightarrow \tau^{q}{ }_{0} D_{t}^{q}$, where ${ }_{0} D_{t}^{q}$ is the fractional order derivative, most simply described by the Grünwald algorithm

$$
\begin{aligned}
{ }_{a} D_{t}^{q} f(t)=\lim _{N \rightarrow \infty} & \left\{\frac{\left[\frac{t-a}{N}\right]^{-q}}{\Gamma(-q)} \sum_{j=0}^{N-1} \frac{\Gamma(j-q)}{\Gamma(j+1)}\right. \\
& \left.\times f\left(t-j\left[\frac{t-a}{N}\right]\right)\right\} .
\end{aligned}
$$

The traditional integer order calculus can be shown to be a subset of the fractional order calculus, i.e. the fractional calculus is to integer order calculus as the real number line is to the integers (Oldham and Spanier, 1974). The definition of Eq. (19) applies to all orders of exponent $q$, both positive (derivative-like) and negative (integral-like). Note also that the fractional "differ-integral", as it is sometimes called, is a linear operator; superposition applies and no new frequency components are created. It produces power-law behavior for $q \notin N$ and exponential behavior for $q \in N$. The definition can also be seen as an integral transform with a power-law kernel. Using this definition, we perform an inverse Laplace transform of Eq. (15) to get

$$
\begin{gathered}
\left(1+\tau_{0}^{\delta} D_{t}^{\delta}\right) \mathbf{D}(t)=\left(\varepsilon_{\mathrm{S}}+\varepsilon_{\infty} \tau^{\delta}{ }_{0} D_{t}^{\delta}+\varepsilon_{\mathrm{C}} \tau^{\beta}{ }_{0} D_{t}^{\beta}\right. \\
\left.+\varepsilon_{\mathrm{C}} \tau_{0}^{v} D_{t}^{v}\right) \varepsilon_{0} \mathbf{E}(t),
\end{gathered}
$$

where $\varepsilon_{0}$ is the permittivity of free space and the $\varepsilon_{k}$ are the relative permittivities described above.

One of the most significant predictions of the power-law form of the constitutive equation is that the decay currents depend significantly on the history of the applied field. To test whether this 
constitutive equation would actually predict the power-law behavior of long-time scale dielectric relaxation, the values obtained from curve fitting of the spectroscopic measurements were entered into Eq. (20) and the resulting fractional difference equation was numerically integrated over a simulated experiment of more than an hour's duration. The first run consisted of the sample being short circuited for more than $12 \mathrm{~h}$, and then subjected to a $100 \mathrm{~V}$ charging pulse for $1 \mathrm{~h}$, and then shorted. The second run repeated the first, but with a simulated a charging pulse lasting $10 \mathrm{~min}$. The third run simulated a charging pulse of $100 \mathrm{~s}$. In each case, the numerical integration time step was larger than the time scale of the resonance, and so the resonance was ignored.

The results of the simulation were compared with actual experiments following the same protocol using the apparatus of Fig. 10. Since the samples were piezoelectric, several $\mathrm{cm}$ of mechanical insulation were required to keep vibration-induced noise to a minimum. In Fig. 11, we compare the predicted and measured transient response after dropping the voltage to zero at the end of the charging pulse.

Integrating the relaxation current curve over all time predicts that, to within the resolution of the model, all of the charge that is accumulated in/ across the sample during the charging cycle is returned in the reverse current. In this sense, PVDF appears to be a perfect insulator, even though the currents never go to zero. This long phase delay leads to hysteresis and energy loss, although charge does not actually "flow through" the material as it would in an ideal conductor.

We see that the data obtained from steady state ac spectroscopic measurements over time scales of

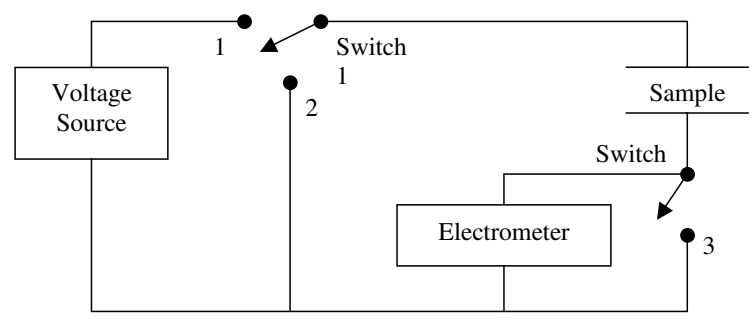

Fig. 10. Schematic of the experimental setup for measuring the transient response.

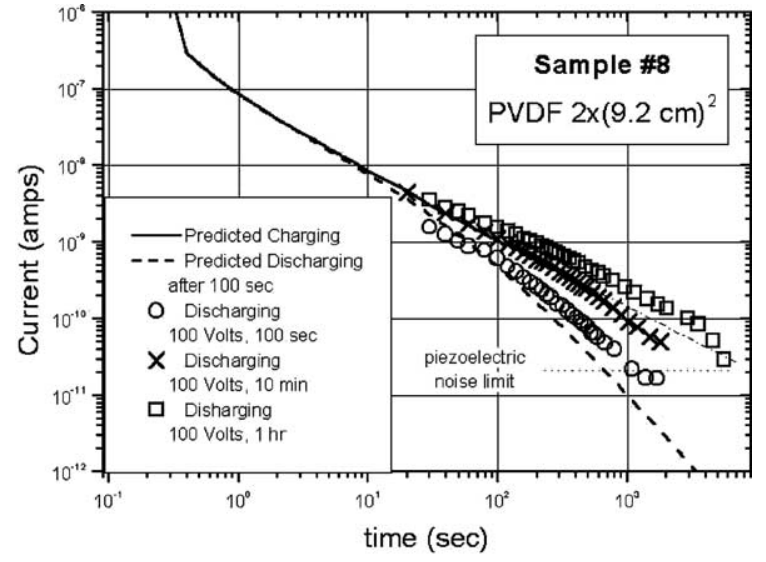

Fig. 11. Measured discharge currents for a sample of $28 \mu \mathrm{m}$ PVDF. Solid and dashed lines are predicted values, symbols are measured data.

$50 \mathrm{~ms}$ and shorter can predict the transient behavior for up to an hour or more with considerable accuracy. This makes a strong case that the response is fundamentally power-law. It is moreover startling that the numerical simulation can simply skip over the time scale of the resonance effect.

\section{Discussion and conclusions}

A program of static, creep and dynamic oscillatory tests performed under various load and temperature conditions was used to determine the mechanical response of PVDF. From our results, it is clear that PVDF can be characterized as an orthotropic, time-dependent material. There are significant differences between the material responses in two in-plane directions (parallel and perpendicular to the aligned molecular chains of the polarized polymer). Although the elastic moduli in both material directions are practically identical, other mechanical characteristics exhibit substantial differences between the two material directions.

Creep and dynamic oscillatory tests of thin films at room temperature show that the linear viscoelastic theory accurately represents the timedependent response of PVDF, if the applied stresses remain below certain limits. Specifically, at 
the stress levels below $57 \%$ of the yield stress in material direction 1 , and at stresses below $76 \%$ of the yield stress in material direction 2, the creep response can be treated as stress independent. It follows that, within these limits, the constitutive equations of linear viscoelastic theory based on the Boltzmann superposition principle can be used to represent the time-dependent response of PVDF. Beyond these stress limits, a nonlinear viscoelastic constitutive law is required to describe the creep properties.

The time-dependent material characteristics of PVDF in both material directions were characterized analytically. The creep compliances of PVDF were represented in the form of a power law. The relaxation functions of the material in both material directions were obtained in the form of infinite series involving gamma functions. Both creep and relaxation characteristics indicate that the time-dependent effects in the material behavior of PVDF are more significant in the direction parallel to the aligned molecular chains of the polymer.

The dynamic mechanical properties of PVDF thin films were characterized by the storage and loss elastic moduli, and storage and loss compliances, and these have been determined experimentally as functions of temperature and frequency. Dynamic tests of PVDF thin films indicate that, at temperatures ranging from 25 to $81{ }^{\circ} \mathrm{C}$, the polymer properties satisfy the temperature-frequency correspondence principle. Within these limits PVDF can be treated as a thermorheologically simple viscoelastic material.

We have so far studied the frequency and time dependent behavior of the mechanical and electrical losses independently. The conditions for these tests were not strictly equivalent: the mechanical measurements were made under mechanically clamped and electrically open boundary conditions, while the electrical measurements were done under mechanically free, electrically closed conditions. Nonetheless, both sets of measurements displayed the same type of power-law creep in displacement or polarization. The long-time scale exponents were consistent; 0.107 and 0.085 in the mechanical case and 0.085 in the electrical case. We point out that the minus sign on the value of the electrical exponent $\beta$, as compared to that of the mechanical exponents $\alpha_{n}$, is due to a difference in definitions. In both cases the power-law behavior introduces a frequencyindependent phase shift resulting in energy loss over all relevant time scales.

In this regard, a logical next step might be to build a model for mechanical creep in fractional calculus form. From there one might begin to see how the long-time behavior of the mechanical response corresponds to that of the electrical response in the piezoelectric regime. A clue that the two are intimately related can be seen in Fig. 12. In this experiment, a bellows actuator was constructed from the $28 \mathrm{~mm}$ PVDF sheets (Bohannan et al., 2000; Mooibroek, 1998). A continuous 200 $\mathrm{V}$ was applied and the displacement without mechanical load was measured over time. The actuator exhibited a displacement creep analogous to that under mechanical loading. It is not yet clear whether the exponents characterizing the longtime behaviors are the same in these two cases.

It is conceivable that some mechanical and dielectric relaxation responses of piezoelectric solids are interrelated. In the literature, a point of view has been expressed that both mechanical and dielectric relaxation result from the same molecular mechanisms (Lakes, 1998). Coupling between mechanical and piezoelectric relaxation in twophase piezoelectric media has been discussed by $\mathrm{Li}$ and Dunn (2001).

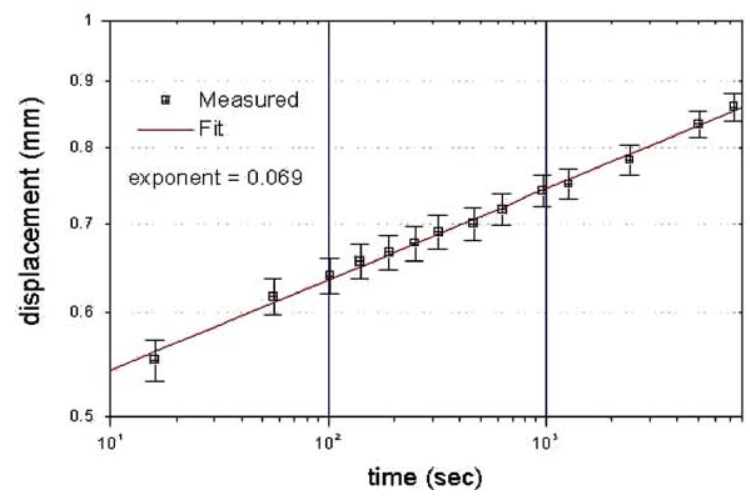

Fig. 12. Change in actuator displacement under constant application of $200 \mathrm{~V}$. 
Further to the studies reported in this paper, we hope to conduct a series of electromechanical measurements that are consistent with the boundary conditions that prevail in most applicationsthat is, mechanically clamped and electrically closed. Of particular interest is the extent to which the electrical relaxation response is related to the $n=1,2$ directions of the mechanical response. Perhaps two long-time-scale mechanisms must be included in the electrical equivalent circuit model to account for coupling to the two different mechanical responses. Such tests would help with the construction of dynamical models that can incorporate creep, hysteresis, and other phenomena associated with the control of piezoelectric materials in applications.

\section{References}

Arisawa, H., Yano, O., Wada, Y., 1981. Dielectric loss of poly(vinylidene fluoride) at low temperatures and effect of poling on the low temperature loss. Ferroelectrics 32, 39-41.

Bohannan, G.W., Schmidt, V.H., Conant, R.J., Hallenberg, J., Nelson, C., Childs, A., Lukes, C., Ballensky, J., Wehri, J., Tikalsky, B., McKenzie, E., 2000. Piezoelectric polymer actuators in a vibration isolation application. Proc. SPIE 3987, 331-342.

Cole, S., Cole, R., 1941. Dispersion and absorption in dielectrics. J. Chem. Phys. 9, 341-351.

Davis, G.T., Broadhurst, M.G., Lovinger, A.J., Furukawa, T., 1984. Hysteresis in copolymers of vinylidene fluoride and trifluoroethylene. Ferroelectrics 57, 73-84.

Ferry, J.D., 1980. Viscoelastic Properties of Polymers. John Wiley \& Sons, New York.

Furukawa, T., Date, M., Fukada, E., 1980. Hysteresis phenomena in polyvinylidene fluoride under high electric field. J. Appl. Phys. 51, 1135-1141.

Furukawa, T., Johnson, G.E., Bair, H.E., Tajitsu, Y., Chiba, A., Fukada, E., 1981. Ferroelectric phase transition in a copolymer of vinylidene fluoride and trifluoroethylene. Ferroelectrics 32, 61-67.
Gookin, D.M., Jacobs, E.W., Hicks, J.C., 1984. Correlation of ferroelectric hysteresis with ferroelastic hysteresis in polyvinylidene fluoride. Ferroelectrics 57, 89-98.

Holloway, F.C., 1997. Material characterization of poly(vinylidene fluoride), a thin film piezoelectric polymer. MS thesis, Montana State University, Bozeman.

Kanwal, R.P., 1971. Linear Integral Equations. Academic Press, New York.

Kawai, H., 1969. The piezoelectricity of poly(vinylidene fluoride). Jpn. Appl. J. Phys. 8, 975-976.

Koga, K., Ohigashi, H., 1986. Piezoelectricity and related properties of vinylidene fluoride and trifluoroethylene copolymers. J. Appl. Phys. 59, 2142-2151.

Lakes, R.S., 1998. Viscoelastic Solids. CRC, Boca Raton.

Landau, L., Lifshitz, E., 1960. Electrodynamics of Continuous Media, A Course in Theoretical Physics, vol. 8. Pergamon Press, Oxford.

Li, J.Y., Dunn, M.L., 2001. Viscoelectroelastic behavior of heterogeneous piezoelectric Solids. J. Appl. Phys. 89 (5), 2893-2903.

Menard, K.P., 1999. Dynamic Mechanical Analysis. CRC Press, New York.

Mooibroek, M., 1998. Vibration control by piezoelectric actuators, Technical Report, Utrecht University and Montana State University.

Nonnenmacher, T., Glockle, W., 1991. A fractional model for mechanical stress relaxation. Phil. Mag. Lett. 64, 89-93.

Oldham, K., Spanier, J., 1974. The Fractional Calculus. Academic Press, New York.

Osaki, S., Kotaka, T., 1981. Electrical properties of form III poly(vinylidene fluoride). Ferroelectrics 32, 1-11.

Roh, Y., Varadan, V.V., Varadan, V.K., 2002. Characterization of all the elastic, dielectric, and piezoelectric constants of uniaxially oriented poled PVDF films. IEEE Trans. Ultrason. Ferroelectr. Freq. Control 49/6, 836-847.

Vinogradov, A.M., 2002. Piezoelectricity in Polymers. Encyclopedia of Smart Materials, vol. 2. John Wiley \& Sons, Inc.. pp. 780-792.

Vinogradov, A.M., Holloway, F., 1999. Electro-mechanical properties of the piezoelectric polymer PVDF. Ferroelectrics 226, 169-181.

Wang, H., Zhang, Q.M., Cross, L.E., 1993. Piezoelectric, dielectric, and elastic properties of poly(vinylidene fluoride/ trifluoroethylene). J. Appl. Phys. 74, 3394-3398.

Wineman, A.S., Rajagopal, K.R., 2000. Mechanical Response of Polymers. Cambridge University Press, Cambridge. 